\title{
Magnetic Field Imaging by Scanning Magnetoresistance Microscope with MR Cantilever
}

\author{
M. Nakamura, T. Takezaki, K. Sueoka, and K. Mukasa \\ Graduate School of Engineering, Hokkaido University, Kita-13 Nishi-8, Kita-ku, Sapporo, 060-8628
}

We developed a micro-fabricated cantilever with an MR sensor (MR cantilever) for use as a scanning magnetoresistance microscope (SMRM). Using a four-point MR cantilever with good signal-to-noise ratio, we have succeeded in imaging the stray field distribution of a magnetic garnet sample. Since the magnetic spatial resolution of the SMRM depends only on the physical dimensions of the MR element, the $5-\mu \mathrm{m}$ by $1-\mu \mathrm{m}$ MR element used in this study provided a submicronmeter spatial resolution in one direction of the SMRM.

Key words: magnetoresistance (MR), SMRM, SPM, cantilever, garnet, magnetic imaging

\section{Introduction}

A number of techniques based on scanning probe microscopy (SPM) have been developed to measure magnetic fields emanating from sample surfaces. Magnetic force microscopy (MFM), which employs a sharp tip coated with a layer of magnetic material, is one example of SPM for magnetic imaging. A combination of SPM and various kinds of magnetic sensors, such as superconducting quantum interference devices (SQUID) ${ }^{1)}$, Hall probes ${ }^{2}$, and magnetoresistive (MR) sensors ${ }^{3)}$, provides quantitative measurement of the magnetic fields from the samples with high spatial resolution. A micro-cantilever equipped with an MR sensor creates a scanning magnetoresistance microscope (SMRM) that can be operated with high field sensitivity at ambient conditions. In the pioneering work on an SMRM reported by Yamamoto et al. $^{3-7)}$, available MR read/write head in contact with sample surfaces was used as a magnetic probe. Therefore they did not have any mechanism to maintain a constant separation between the sample and probe. That means the sample they could observe was limited to flat a flat configuration. Recently, we proposed a new SMRM system employing a cantilever with an MR sensor (we call it an "MR cantilever"), which can precisely control the vertical position of the cantilever during magnetic field measurement. By fabricating and characterizing simple MR cantilevers that we designed, we demonstrated that our MR cantilevers make it possible to obtain topographic and magnetic information simultaneously. We also demonstrated that observed magnetic field images can be labeled quantitatively ${ }^{8}$. Furthermore, to improve sensitivity and reliability of the
MR cantilever, we designed and fabricated a new MR cantilever ${ }^{9}$.

In this paper, we report an application of a four-point MR cantilever for imaging maze domain patterns on magnetic garnet thin films. We also discuss the spatial resolution of the MR cantilever.

\section{Fabrication process}

Fig. 1 shows the fabrication process flow for making the MR cantilever. We fabricated our MR cantilevers using electron beam (EB)- and photo-lithographic techniques, liftoff, dry, wet etching, and bulk micromachining. The fabrication procedure consists of the following steps: (1) photolithography and dry etching to form cantilever shapes on the top, and windows on the bottom, of the wafer, (2) EB lithography and liftoff to make an MR sensor including an MR element and lead electrodes and bonding pads, (3) $\mathrm{KOH}$ wet etching to remove the remaining $\mathrm{Si}$ substrate, (4) wet etching of the $\mathrm{SiO}_{2}$ etch stopping layer for $\mathrm{KOH}$ wet etching to release the cantilevers from the substrate, and (5) removing the layer

(a)
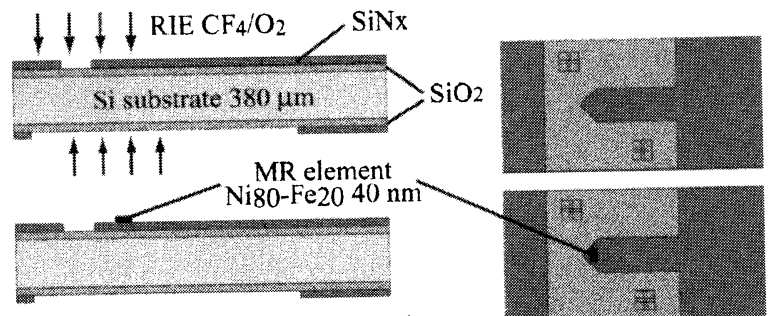

(b)

(c)

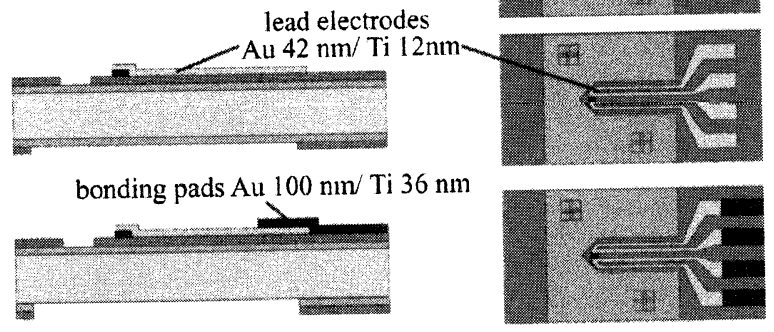

(d)
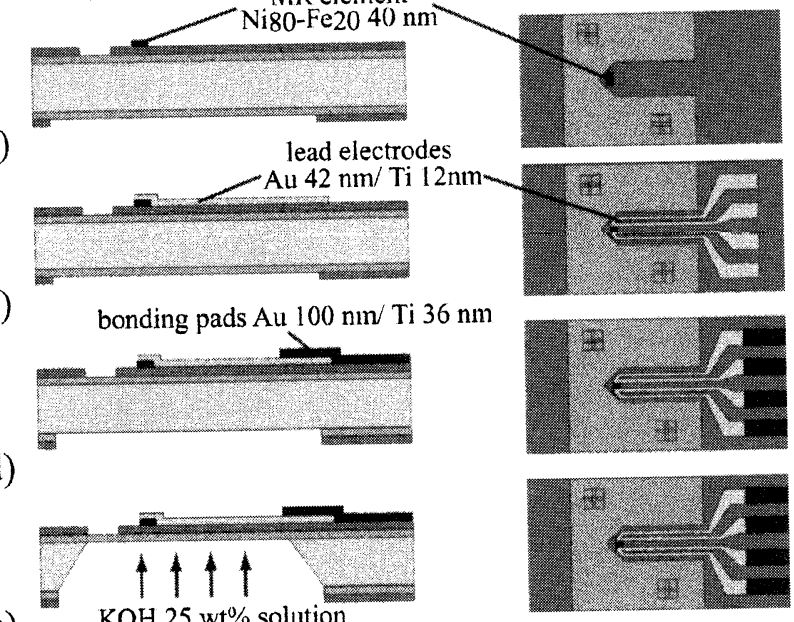

(e)

(f)
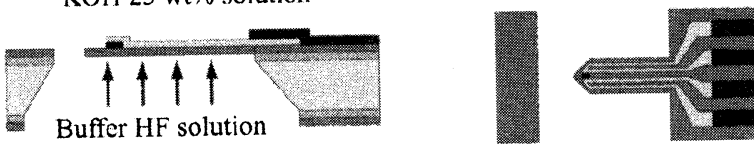

Fig. 1 Fabrication process flow for MR cantilever. 


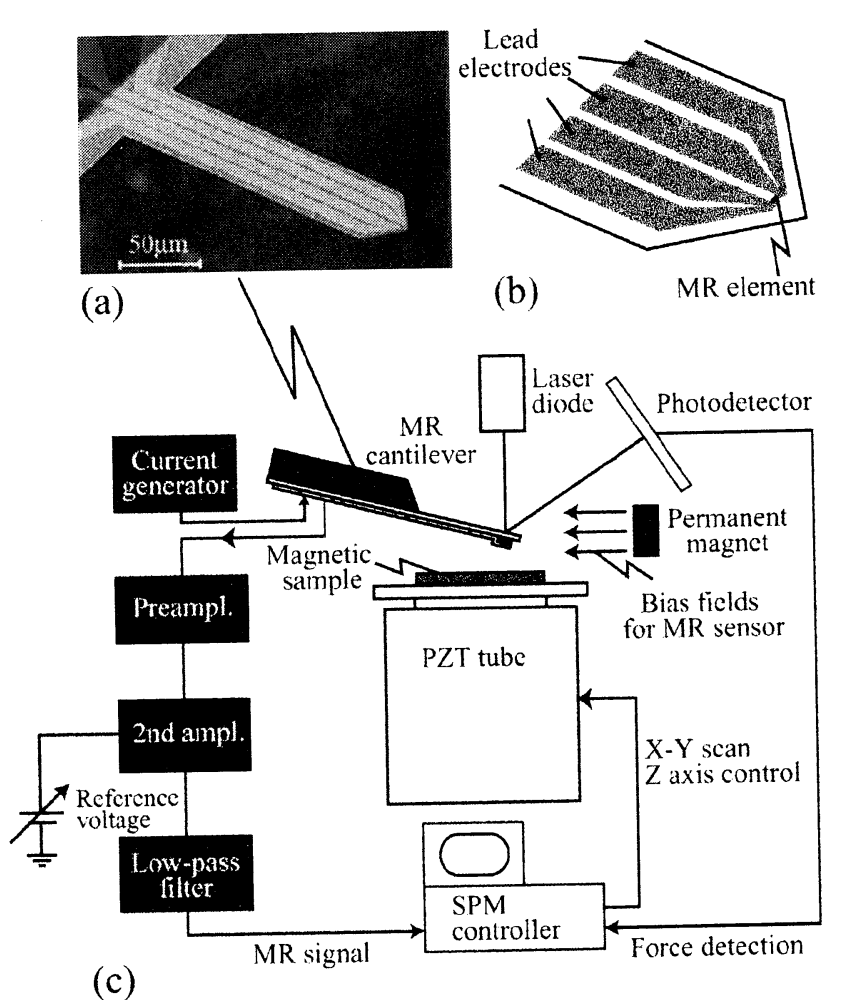

Fig. 2 (a) Scanning electron micrograph of a typical MR cantilever; (b) apex of the MR cantilever; (c) schematic diagram of the SMRM system.

protected from wet etching. The fabrication process for the MR cantilever has been described in detail elsewhere ${ }^{8)-11)}$. In this study, in order to reduce the residual stress of the $\mathrm{Au} / \mathrm{Ti}$ electrodes, we fabricated the electrodes separately from the bonding pads as shown in Figs. 1(c) and 1(d), and reduced the thickness of the electrodes on the cantilever. The thickness of the lead electrodes and bonding pads are $\mathrm{Au}(42 \mathrm{~nm}) / \mathrm{Ti}(12 \mathrm{~nm})$ and $\mathrm{Au}(100$ $\mathrm{nm}) / \mathrm{Ti}(36 \mathrm{~nm})$ respectively.

Fig. 2 shows a scanning electron microscopy (SEM) image, a schematic illustration of a typical MR cantilever, and a schematic of the SMRM measurement system. As shown in Fig. 2, at the apex of the cantilevers, the MR element is connected with four $\mathrm{Au} / \mathrm{Ti}$ electrodes. The four-point sensor configuration has the advantages of negligible resistance of the lead electrodes and of improved the signal-to-noise ratio $(\mathrm{S} / \mathrm{N})$ as a result of reducing the total resistance of the MR sensor. The dimensions of the MR element used in this study are $5 \mu \mathrm{m}$ long and $1 \mu \mathrm{m}$ wide. Since the MR sensor is anisotropic, the MR element must be biased magnetically in order to operate the MR sensor linearly with respect to external fields. We place a permanent magnet near the MR cantilever and apply a bias magnetic field of about $100 \mathrm{Oe}$ at the position of the MR element. The MR cantilever is mounted on the cantilever holder at a tilt angle of $13^{\circ}$ with respect to the sample surface. The sharpened apex of the cantilever serves as an AFM probe and obtains topographic information for the samples. We apply the MR sensor through a constant current of $0.8 \mathrm{~mA}$ and detect the change in output voltage depending on the external field. The resistance of the MR sensor is about 34 $\Omega$, and its MR ratio is about $1.2 \%$. In this SMRM experiment, the MR element is positioned at a height of about $1 \mu \mathrm{m}$ above the sample surface. The detection direction of the SMRM is perpendicular to the long axis of the MR element and parallel to the plane of the MR cantilever.

\section{Results and discussion}

Fig. 3 (a) shows an example of $30 \mu \mathrm{m}$ X $30 \mu \mathrm{m}$ SMRM images of the garnet thin film. The contrast of this image corresponds to the strength of the detectable direction component of the stray field from the garnet surface. We operate the SMRM in contact AFM mode, and get the simultaneous AFM/ SMRM images (the AFM image is not shown in Fig. 3). The SMRM can image clearly the typical domain structure (i.e. maze domain structures) of the garnet sample. We observed the same sample using the commercial MFM (not shown in this paper), and confirmed that the period of the domain stripe of this garnet is approximately $4 \mu \mathrm{m}$. Figs. 3(b) and (c) show cross-sectional profiles in the direction of the arrows $A$ and $B$ in Fig. 3(a), respectively. In the area around the arrow $A$, the domain configuration of the garnet is vertically striped pattern, it is expected that the field in the horizontal direction, which is parallel to the detectable direction of the SMRM, is dominant, so that the response of the MR sensor is large. And since the horizontal dimension of the MR element is $1 \mu \mathrm{m}$, which is smaller than the period of the domain stripe, the SMRM can achieve the sub-micron resolution in this direction. In contrast, in the arca shown as the arrow $\mathrm{B}$, the domain is horizontally striped pattern, the field in the horizontal direction is weak. And since the vertical dimension $(5 \mu \mathrm{m})$ is rather large compared with the period of domain, the deconvolution of the signal (so called "off-track response") may be occurred, so that the signal amplitude in this direction becomes smaller than that in the direction of the arrow A. Because the spatial resolution of the magnetic imaging depends mainly on the physical size of the MR element, it is a reasonable result that the spatial resolution is worse along the vertical direction than along the horizontal direction.

These cross-sectional profiles indicate also that the output voltage is saturated at all peak points. The reason for the saturation of the MR output is that the bias field for the MR element is not enough to operate the MR sensor properly. The transfer curve of the MR sensor is shown in Fig. 4. In our SMRM system, since the permanent magnet is used to bias the MR element perpendicularly, it is difficult to control the strength of the bias field arbitrarily. At the operating point (the bias field is about $100 \mathrm{Oe}$ ) the 


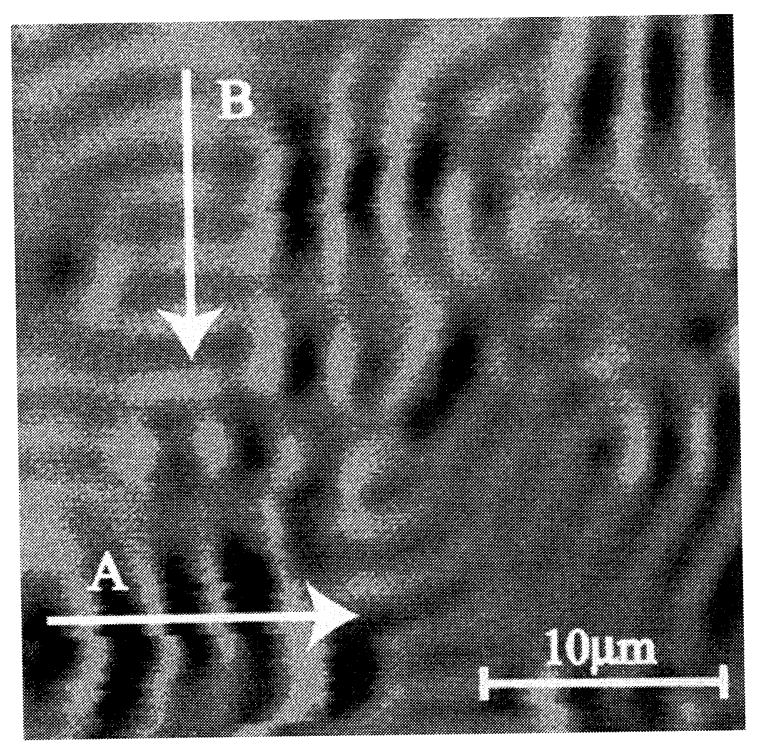

(a) $-0.0147 \mathrm{~V}$

$0.0060 \mathrm{~V}$
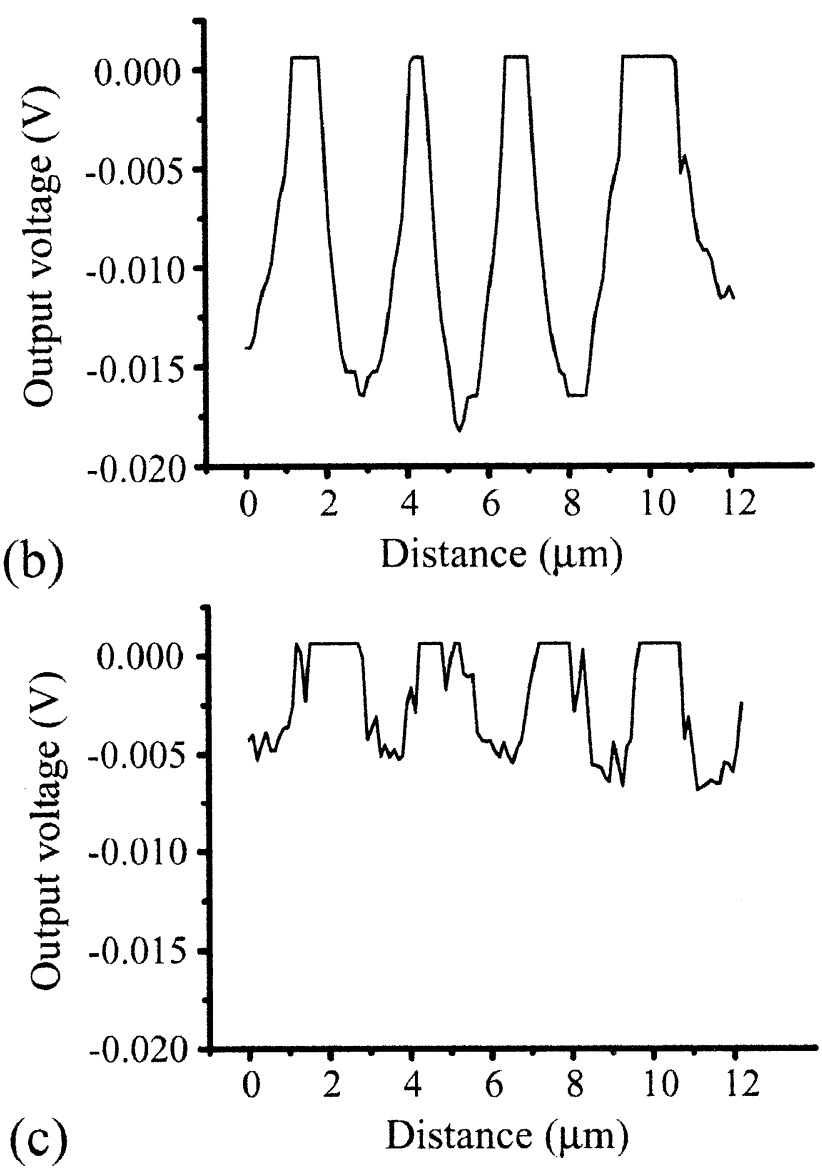

Fig. 3 Image (a) is the SMRM image of the garnet sample; (b) and (c) are cross-sectional profiles in the directions of arrows A and B in (a), respectively. These line profiles indicate that the horizontal resolution is better than the vertical. The output signal is saturated at several peak points.

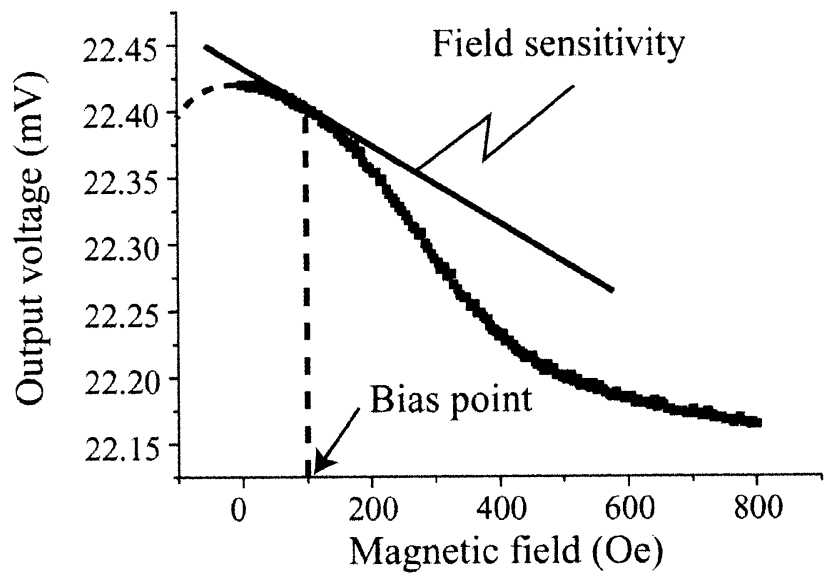

Fig. 4 Transfer curve of the MR sensor at a bias point. The solid line indicates the deviation of the curve (i.e., the sensitivity of the MR sensor) at a bias point of about 100 Oe.

is very narrow, and the field sensitivity (i.e. the normalized derivative of the transfer curve at a bias point) is also very low $(\sim 8.9 \mu \mathrm{V} / \mathrm{V} / \mathrm{Oe})$. When there is a large external magnetic field in the direction opposite that of the bias field, the MR output is saturated. If there is a larger external field, the MR output begins to be reduced through the maximum point of the transfer curve. By applying the proper bias field strength $(\sim 300 \mathrm{Oe})$, we can improve the field sensitivity of SMRM to about $26.8 \mu \mathrm{V} / \mathrm{V} / \mathrm{Oe}$. However, if the bias field becomes too large, it can affect the domain structure of the sample and the distribution of the stray field emanating from the sample. In order to improve the properties of MR sensors further, we need to optimize the dimensions of the MR element for aspect ratio (length/width). A spin valve sensor having a self-bias structure is another candidate for a cantilever magnetic sensor to make the SMRM system more sensitive and functional.

Finally, we wish to discuss the upper bound of SMRM spatial resolution. Since miniaturizing the MR element degrades the sensitivity of the SMRM because of the reduction in resistance, we examined the relationship of the SMRM signal-to-noise ratio (SNR) to the dimensions of the MR element. For simplicity, we assume that the sensitivity of the MR element is constant if the aspect ratio of the MR element does not change. This assumption means that the magnetization in the MR element can rotate uniformly with respect to external fields. If the MR element is thin enough compared with the in-plane dimension, the shape of the transfer curve depends only on the anisotropic field that is determined by the aspect ratio of the MR element. The signal is assumed as the variation in MR ratio in the range of the linear region with respect to the external magnetic field of the transfer curve.

$$
\text { Signal }=S \cdot H \cdot \rho \frac{L}{W t} \cdot i \cdot W t=S \cdot H \cdot \rho \cdot i \cdot L,
$$

where $S$ is the sensitivity of the MR sensor; $H$ is dynamic range of the MR sensor within which the MR output is 


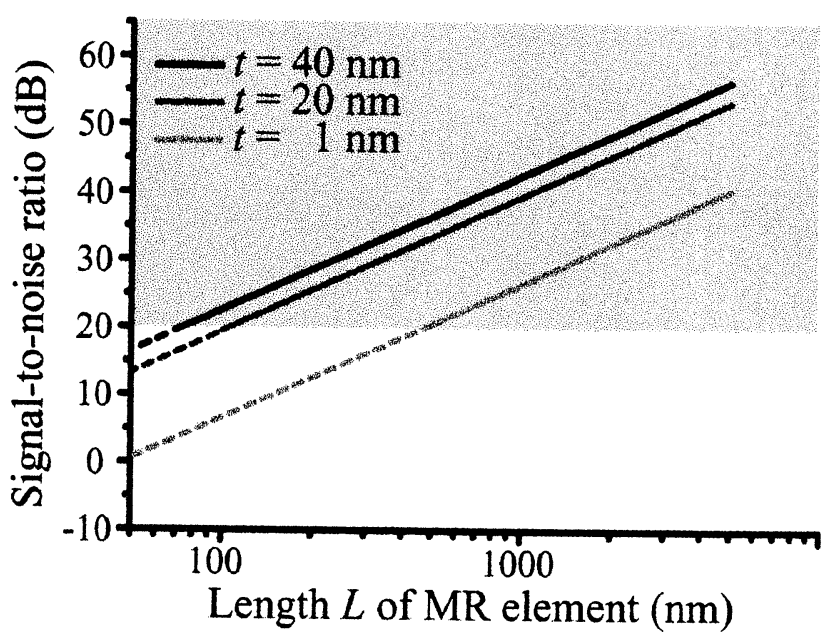

Fig. 5 Signal-to-noise ratio of the SMRM versus the length of the MR element.

linear with respect to the external field; $\rho$ is the resistivity of the MR element; $L, W$, and $t$ are the length, width, and thickness of the MR element, respectively; and $i$ is the allowable current density limited by electromigration effect. The dominant noise component is found to be the Johnson noise,

$$
\text { Noise }=\sqrt{4 k T R \Delta f}=\sqrt{4 k T \Delta f \rho \frac{L}{W t}}=\sqrt{4 k T \Delta f \rho \frac{A}{t}},
$$

where $k, T$, and $\Delta f$ are the Boltzmann constant, temperature, and bandwidth, respectively. $A$ is the aspect ratio of the MR element $(A=L / W)$. Fig. 5 shows the signal-to-noise ratios versus $L$ for several values of thickness, where $S=26.8 \mu \mathrm{V} / \mathrm{V} / \mathrm{Oe}, H=200 \mathrm{Oe}$ obtained from the transfer curve in Fig. 4. The resistivity of the permalloy $\rho=20 \mu \Omega \mathrm{cm}, i=10^{6} \mathrm{~A} / \mathrm{cm}^{2}, k=1.38 \times 10^{-23}$ $\mathrm{J} / \mathrm{K}, T=300 \mathrm{~K}$, and $\Delta f=10 \mathrm{kHz}$. The aspect ratio of the element is the same value of this experimental arrangement: $A=5$. When the size of the MR element is miniaturized holding the aspect ratio constant, the resistance of the MR element becomes smaller, so the SMRM output signal is also reduced. On the other hand, because the thickness of the element is constant and the Johnson noise does not depend on the aspect ratio $A$, the signal-to-noise ratio decreases with the in-plane dimension of the MR element. We assumed that the lower limit of the signal-to-noise value, which is enough to operate the MR sensor as a magnetic probe of the SMRM, is $20 \mathrm{~dB}$. Under these conditions, we estimated that the smallest length, $L$, of the MR element is about $70 \mathrm{~nm}$ at $t=40 \mathrm{~nm}$, i.e., the limit of the spatial resolution of the SMRM is about $70 \mathrm{~nm}$ in one direction and a few tens of nanometers in the other. In order to obtain better spatial resolution, ferromagnetic materials having a higher MR ratio must be used as the MR elements, or high-sensitivity sensors, such as spin valve sensors, must be applied to the SMRM.

\section{Conclusion}

We succeeded in imaging the stray magnetic field from garnet sample using a four-point $\mathrm{MR}$ cantilever. The SMRM image exhibits good signal-to-noise ratio and high spatial resolution (sub- $\mu \mathrm{m}$ order). Based on simple estimation of the signal-to-noise ratio, the SMRM using AMR sensors potentially has a spatial resolution of less than $100 \mathrm{~nm}$. In the experimental configuration reported here, since the small bias field of the MR element leads to saturating the MR output voltage, it is difficult to transfer the output voltage into an external magnetic field in saturated regions. These problems can be solved by using spin valve sensors or properly designed anisotropic MR sensors (for example, dual MR sensors, or soft adjacent layer biased MR sensors).

\section{References}

1) J. R. Kirtley, M. B. Ketchen, K. G. Stawiasz, J. Z. Sun, W. J. Gallagher, S. H. Blanton, and S. J. Wind: Appl. Phys. Lett. 66, 1138 (1995).

2) A. M. Chang, H. D. Hallen, L. Harriott, H. F. Hess, H. L. Kao, J. Kwo, R. E. Miller, R. Wolfe, J. van der Ziel, and T. Y. Chang: Appl. Phys. Lett. 61, 1974 (1992).

3) S. Y. Yamamoto and S. Schultz: Appl. Phys. Lett. 69, 3263 (1996).

4) S. Y. Yamamoto, D. C. Vier, and S. Schultz: IEEE Trans. Magn. 32, 3410 (1996)

5) S. Y. Yamamoto and S. Schultz: J. Appl. Phys. 81, 4696 (1997).

6) S. Y. Yamamoto, S. Schultz, Yun Zhang, and H. Neal Bertram: IEEE Trans. Magn. 33, 891 (1997).

7) S. Y. Yamamoto, R. O'Barr, S. Schultz and A. Scherer: IEEE Trans. Magn. 33, 3016 (1997).

8) M. Nakamura, M. Kimura, K. Sueoka, and K. Mukasa: Appl. Phys. Lett. 80, 2713 (2002)

9) M. Nakamura, K. Sueoka, and K. Mukasa: Trans. Magn. Soc. Jpn. 2, 7 (2002).

10) M. Nakamura, M. Kimura, K. Sueoka, and K. Mukasa: $J$. Magn. Soc. Jpn. 24, 771 (2000).

11) M. Kimura, M. Nakamura, K. Sueoka, and K. Mukasa: $J$. Magn. Soc. Jpn. 25, 1079 (2001).

(Received Oct. 7, 2002; Accepted Jan. 17, 2003) 